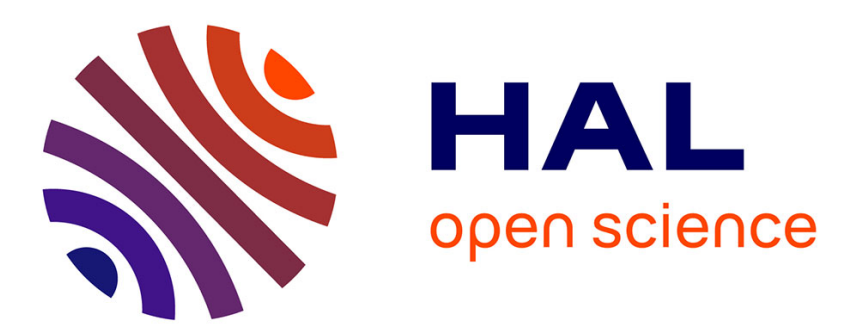

\title{
Statistical aspects in crack growth phenomena: how the fluctuations reveal the failure mechanisms \\ Laurent Ponson
}

\section{To cite this version:}

Laurent Ponson. Statistical aspects in crack growth phenomena: how the fluctuations reveal the failure mechanisms. International Journal of Fracture, 2016, 201 (1), pp.11-27. 10.1007/s10704-016-0117-7 . hal-01377003

\section{HAL Id: hal-01377003 \\ https://hal.sorbonne-universite.fr/hal-01377003}

Submitted on 6 Oct 2016

HAL is a multi-disciplinary open access archive for the deposit and dissemination of scientific research documents, whether they are published or not. The documents may come from teaching and research institutions in France or abroad, or from public or private research centers.
L'archive ouverte pluridisciplinaire HAL, est destinée au dépôt et à la diffusion de documents scientifiques de niveau recherche, publiés ou non, émanant des établissements d'enseignement et de recherche français ou étrangers, des laboratoires publics ou privés. 


\title{
Statistical aspects in crack growth phenomena: How the fluctuations reveal the failure mechanisms
}

\author{
Laurent Ponson
}

\begin{abstract}
Material failure often gives rise to strong fluctuations that reflect on the rough trajectory followed by cracks and on their intermittent dynamics. Understanding the origin of these fluctuations is a major challenge in fracture mechanics since they emerge from the interaction of the cracks with the material microstructure that it still poorly understood. Here, we illustrate through recent studies how the statistical properties of these fluctuations can reveal elementary failure mechanisms taking place at the microstructure scale. The implications of these findings in terms of material characterization and failure analysis is discussed and some promising directions for future investigations are presented.
\end{abstract}

Keywords Statistical approach · crack roughness · velocity fluctuations · disordered materials · scaling properties . fractography

\section{Introduction}

Crack propagation is the central mechanism leading to material failure. However, crack growth phenomena are far from being fully understood. A major challenge underlying fracture problems relates to its multiscale nature: The macroscopic failure behavior of materials are largely governed by microstructural features and processes localized at the crack tip vicinity. Cracks that are efficient stress concentrators exacerbate their impact that can be felt on macroscopic quantities like toughness and crack speed.

For brittle cracks, this strong coupling between length scales has been used as an opportunity to design systems with improved failure properties, as patterning their structure at the small scale can enhance their resistance at the large scale (Xia et al., 2012; Hossain et al., 2014). But when complex dissipative mechanisms come into play, going from

Institut Jean le Rond d'Alembert (UMR 7190), CNRS - Université Pierre et Marie Curie, 75005 Paris, France the small to the large, i.e. from the microscale features of materials to their macroscale failure behavior, remains a challenging task. The multiscale nature of fracture phenomena can then be used as a means to probe local failure mechanisms. Altough they might not be amenable to direct observations due to the small length and time scales involved, their impact on the macroscopic material response may indeed be more easily characterized. A direct manifestation of microscale processes at the large scale is the strongly fluctuating behavior of cracks. The challenge raised by this approach then concerns the interpretation of these fluctuations in term of physical processes.

Extracting meaningful information from fluctuations has been a preferred line of research in various domains, including the physics of condensed matter. As a result, the approaches and concepts developed in these fields have been largely borrowed and applied to fracture problems (Alava et al., 2006; Bonamy and Bouchaud, 2011). A major output of these works has been to establish a connection between the failure of disordered materials and critical phenomena like percolation or depinning transition. In critical phenomena, the system behavior and its fluctuations can be characterized by power laws that emerge from the competition between disorder and long-range interactions. In the context of material failure, the observed power laws emerge from the competition between the microstructural disorder of the material and the elasticity, very often influenced by damage processes. In the following, we will illustrate through some recent research studies how to make sense to these scaling behaviors and use them to identify and characterize elementary crack growth mechanisms. We will focus on two quantities that are ready-made directions of investigation in failure problems, the crack speed and its fluctuations in Section 2 and the crack path and its fluctuations in section 3. The Section 4 discusses promising research directions for future investigations. 

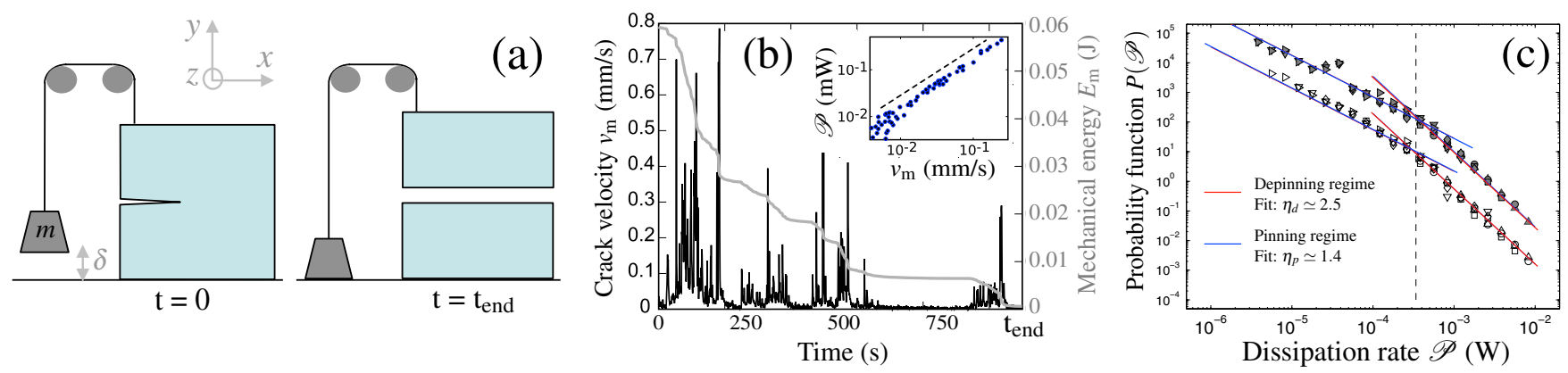

Fig. 1 (a): Isolated system of total energy $E_{\mathrm{tot}}=E_{\mathrm{m}}(t)+E_{\mathrm{f}}(t)$ consistuted of a notched specimen under dead weight loading conditions. Before failure, the energy is stored as potential energy $E_{\mathrm{tot}}=E_{\mathrm{m}}(0)$ that has been fully dissipated in fracture energy $E_{\mathrm{tot}}=E_{\mathrm{f}}\left(t_{\mathrm{end}}\right)$ after failure. (b): Experiments in heterogeneous solids show that this transfer from mechanical into fracture energy proceeds through bursts that can be studied through the variations of the crack velocity $v_{\mathrm{m}}$ that is proportional to the dissipation rate $\mathscr{P}=-d E_{\mathrm{m}} / d t=d E_{\mathrm{f}} / d t$ as shown in inset. (c) Probability density of the dissipation rate. The different symbols correspond to different experimental sampling rates, while both curves correspond to two average crack growth velocities. [Figs. (b) and (c) are courtesy of Barés et al. (2014)]

\section{Statistics of fluctuations in the dynamics of cracks}

\subsection{Experimental observations}

Crack dynamics has been extensively used to investigate failure mechanisms in materials. Here, the emphasis is put on the fluctuations of crack speed that can be either investigated at the local scale, i.e. at some location along the crack front, or at the global scale, through the evolution of the average crack position. Interestingly, this second approach amounts to investigate the rate of dissipated energy through failure. To establish this connection, one considers the isolated system depicted in Fig. 1(a) made of a notched specimen and a dead load pulling on its upper face through frictionless pulleys. The total energy $E_{\mathrm{tot}}=E_{\mathrm{m}}+E_{\mathrm{f}}$ can be partitioned into mechanical and fracture energy. The process of failure proceeds through a transfer of the first contribution into the second one.

In the example depicted in Fig. 1, the initial condition corresponds to a state of the system where the energy is entirely stored in mechanical energy with $\left\{E_{\mathrm{m}}^{0}=E_{\mathrm{tot}}, E_{\mathrm{f}}^{0}=0\right\}$ at $t=0$. On the contrary, once the sample is broken, all the mechanical energy initialy available has been dissipated into fracture so that $\left\{E_{\mathrm{m}}^{\mathrm{end}}=0, E_{\mathrm{f}}^{\mathrm{end}}=E_{\mathrm{tot}}\right\}$ at $t=t_{\text {end }}$. In practice, for more general loading conditions, the total dissipated energy corresponds to the work of the force applied to the sample during the test that reduces well to the potential en$\operatorname{ergy} E_{\mathrm{m}}^{0}=m g \delta$ of the dead load $m$ displaced over the height $\delta$ in the specific example considered here.

To describe this energy transfer, we introduce the elastic energy release rate $G(z, t)$ that measures the decrease $d E_{m}(t)=-\int_{0}^{b} G(z, t) d f(z, t)$ of mechanical energy for an incremental crack advance $d f(z, t)$, where $f(z, t)$ provides the crack length in $z$ at time $t$ and $b$ denotes the sample width along the $z$-axis. Wherever the crack propagates, $G(z, t)$ is equal to the rate of energy dissipated or fracture energy $G_{\mathrm{c}}$, so that the mechanical energy released compensates the energy dissipated $d E_{f}(t)=\int_{0}^{b} G_{\mathrm{c}} d f(z, t)$. It follows that the transfer rate

$$
\mathscr{P}(t)=\frac{d E_{s}}{d t}=-\frac{d E_{m}}{d t}=\int_{0}^{b} G(z, t) \frac{\partial f}{\partial t} d z \simeq b G_{\mathrm{c}} v_{\mathrm{m}}(t)
$$

is proportional to the average crack growth velocity $v_{m}$. This linear relation has been tested experimentally in Barés et al. (2014) and is shown in the inset of Fig. 1(b). The proportionality constant provides the material fracture energy $G_{\mathrm{c}}$.

Interestingly, in disordered solids, the dissipation rate is far from being constant in time. Instead, it displays a strong intermittency characterized by bursts of failure activities, as exemplified in Fig. 1(b) for an artificial rock made of sintered PMMA beads. This observation contrasts with the smooth variation of the displacement imposed to the fracturing specimen that produces a smoothly varying rate $d W / d t$ of mechanical energy injected into the system. To reconcile these two different dynamics, we separate the mechanical energy $E_{\mathrm{m}}=W+E_{\mathrm{el}}$ into the work of the external force that varies smoothly with time and the elastic energy stored in the sample from which emerges these strong fluctuations. The elastic solid, by storing potential energy and suddenly releasing it through avalanches, acts as a complex filter that drains intermittently the energy flux from the external loading to the crack tip. This stick-slip dynamics is a direct consequence of the disorder nature of the fracturing material.

The velocity signal $v_{\mathrm{m}}(t)$, or equivalently the dissipation rate since $\mathscr{P} \sim v_{\mathrm{m}}$, are natural candidates to disentangle this complex dynamics. Their statistics is investigated in Fig. 1(c) that shows the probability density function of $\mathscr{P}$. The experiments of Barés et al. (2014) show two regimes characterized by power law behaviors with the exponents $\eta_{p} \simeq 1.4$ and $\eta_{d} \simeq 2.5$ at small and large dissipation rates, respectively. The presence of two distinct regimes reflects the unique dynamics of cracks in disordered materials that 


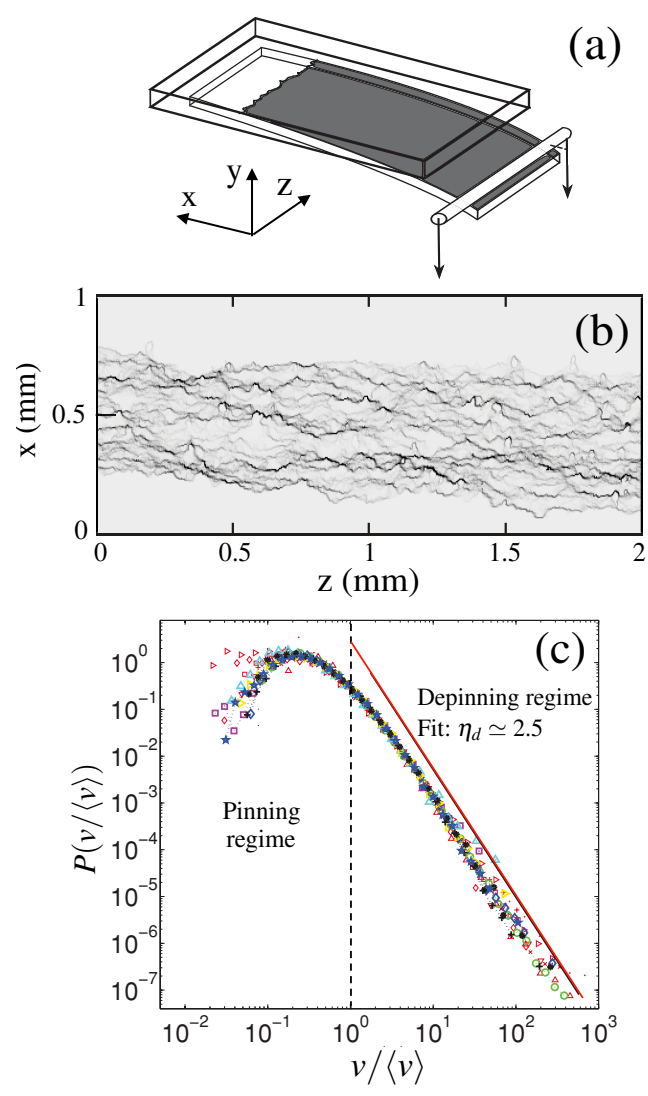

Fig. 2 (a) Sketch of the experimental setup: Two PMMA plates are sintered together, creating a weak plane for the fracture to propagate. (b) Time spent by the front in the different regions of the fracture plane. The dark lines correspond to long waiting times and thus low velocities while the white domains correspond to micro-instabilities and so fast velocities. (c) Distribution of local crack growth velocities. [Courtesy of Måløy et al. (2006)]

is dominated by rare peaks of activity where the dissipation rate is exceptionally large, separated by almost silent periods where the crack speed and so the dissipation rate is much lower.

To identify the physical processes behind these puzzling observations, it is fruitful to observe crack motion at the local scale, where the front interacts with the material microstructure. Figure 2(a) shows an experimental setup designed by Måløy and Schmittbuhl (2001) to explore the local dynamics of crack fronts in disordered solids. In this system, the crack front is confined at the weak interface between two transparent PMMA plates where toughness heterogeneities have been introduced by sandblasting one of the two plates before sintering them together. The complex evolution of the crack recorded at the micrometer scale using a fast camera is illustrated in Fig. 2(b) that shows in grey scale the time spent by the front in the different regions of the fracture plane. The intermittency evidenced at the large scale in Fig. 1(b) is also obvious at the local scale: The black lines visible in Fig. 2(b) that correspond to long wait-
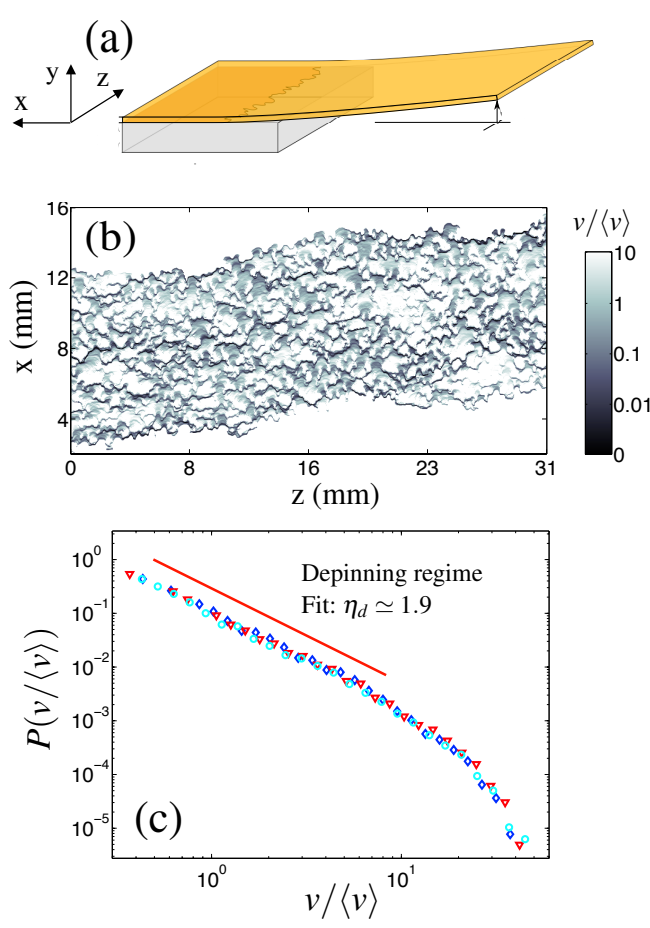

Fig. 3 (a) Sketch of the experimental setup: A thin rigid cantilever is detached from a thick PDMS specimen. The interface where the crack propagates is patterned with randomly distributed obstacles of controlled strength and size. (b) Intermittent dynamics of the crack front in the fracture plane. The dark regions correspond to long waiting times and thus a low velocity while the bright regions correspond to avalanches and so fast velocities. (c) Distribution of local crack growth velocities.

ing time indicate front configurations that have remained trapped by the strongest material heterogeneities. The white regions on the contrary are reminiscent of micro-instabilities during which the front goes rapidly from one stable configuration to another. The coexistence of two antagonistic behaviors is clearly evidenced in the statistics of local crack velocity shown in Fig. 2(c). Their probability density shows two distinct regimes where the small $v<\langle v\rangle$ provides the time spent by the front before escaping from a pinned configuration while the large $v>\langle v\rangle$ characterizes the front dynamics during the so-called avalanches right after depinning and before it gets pinned again in another configuration. Interestingly, the power law behavior observed at the local scale in the depinning regime is characterized by the exponent $\eta_{d} \simeq 2.5$ also measured at the global scale in the experiments of Barés et al. (2014) presented in Fig. 1. How do fluctuations resulting from the pinning of the front by heterogeneities introduced at small scale produce speed variations measurable at the sample scale? This survival of the power law statistics with exponent $\eta_{d} \simeq 2.5$ has been explained by Tallakstad et al. (2013): The central limit theorem that generally ensures Gaussian fluctuations of global velocities as e.g. for front imibibitions (Clotet et al., 2014) breaks 
down here since for large exponents $\eta_{d}>2$, the variance of the local crack speed probability diverges.

Does this imply that cracks systematically display such strong velocity fluctuations at all scales? Not necessarily, because for weak heterogeneities under some specific loading conditions detailed in Roux et al. (2003) and Barés et al. (2013), the dynamics of the crack recovers a continuum like motion as expected for a coarse-grained homogeneous medium.

Interestingly, even in presence of a strong intermittency in the dynamics of the crack, another statistical behavior can be observed. Figure 3(a) depicts an experimental setup where a crack is driven between a transparent PDMS block and a stiff thin plate patterned with randomly located defects of controlled strength and density. This system is inspired from the experiments of Dalmas et al. (2009), Xia et al. (2012) and Chopin et al. (2015) where cracks are pinned by designed obstacles. Similary to Måløy et al. (1992)'s observations [Fig. 2], the front dynamics is also very intermittent, as illustrated by the map of Fig. 3(b) of the front waiting times. However, the scaling behavior of the probability density of local crack speed is characterized by an exponent $\eta_{d} \simeq 1.9$ significantly lower. We will see in the next section devoted to models of crack propagation that this difference reveals two different failure growth mechanisms.

\subsection{Models of crack propagation in disordered materials}

Two types of models have been proposed to describe crack growth in disordered materials. In the first approach, damage processes taking place at the crack tip vicinity are assumed to be localized in a process zone of size $\ell_{p z}$ small with respect to the heterogeneity size $\xi$, as illustrated in Fig. 4(a). This assumption justifies the description of failure processes at a continuum scale through the basic concept of Linear Elastic Fracture Mechanics (LEFM): During crack growth, the rate of elastic energy released compensates the rate of energy dissipated through fracture. Thus, fracture is described as a transfert of the mechanical energy consisting of the elastic energy stored into the loaded specimen and the work of the external force, into fracture energy that consists of the energy dissipated within the crack tip process zone to make the crack propagates. The second type of models takes into account the discrete nature of failure processes by describing crack growth as a succession of discrete failure events in a network of fuses, bonds or beams. Failure takes place when the stress applied locally exceeds a resistance threshold that is randomly distributed among the constitutive elements (Herrmann and Roux, 1990). Contrary to LEFM based model that ensures proper energy conservation during failure, this approach does not rely on thermodynamics principles, and so does not provide any interpretation of the process of crack growth in terms of fracture energy. However, it allows the exploration of complex crack geometries and fracture patterns as the one presented in Fig. 4(b) where crack growth proceeds through the nucleation, growth and coalescence of damage cavities. Both approaches are complementary and apply to different crack propagation problems, as illustrated in the following. Interestingly, a clear connection between both approaches were recently made, as it was shown that discrete models could recover LEFM predictions when investigated at a scale much larger than the process zone size $\ell_{p z}$ (Gjerden et al., 2013).

Continuous models of crack propagation in heterogeneous media builds on two ingredients: The accurate description of the stress field at the vicinity of a distorted crack front (Lazarus, 2011) and the description of the material microstructure at a mesoscale though a heterogeneous field of fracture energy. The first one provides the distribution of crack driving force along the front, prerequisite to predict its evolution within a medium with heterogeneous failure properties. This distribution can generally be obtained explicitly for small front perturbations with respect to straightness, even though larger front perturbations through second order extensions could recently be explored using this approach (Vasoya et al., 2015).

Following this line, Rice (1985)'s formula derived for a semi-infinite planar crack embedded in an infinite elastic medium have been extensively used for investigating the dynamics of cracks in heterogeneous media (Gao and Rice, 1989; Schmittbuhl et al., 1995; Bonamy et al., 2008). It provides the elastic energy release rate distribution

$$
\frac{G(z, t)}{G_{0}}=1+\frac{f_{0}-f(z, t)}{\mathscr{L}}+\frac{1}{\pi} P V \int_{-\infty}^{+\infty} \frac{f(\tilde{z}, t)-f(z, t)}{(\tilde{z}-z)^{2}} d \tilde{z}
$$

as a function of the crack front geometry $f(z)$ defined from a reference straight configuration $f_{0}$ (see Fig 4(a)). ${ }^{1}$ The structural length $\mathscr{L}=-\frac{G_{0}}{d G_{0} / d f_{0}}$ is positive for stable macroscopic loading conditions for which the unperturbed elastic energy release rate $G_{0}$ decays with the unperturbed crack position under fixed loading. This formula actually applies also for a crack lying at the interface between a rigid substrate and an incompressible material (Pindra et al., 2008), so it can be safely applied to the two experimental situations presented previously. Note that formula similar to Eq. (2), but involving different kernels in the integral, can be derived for more specific fracture geometries, e.g. to take into account the finite thickness of the specimen (Legrand et al., 2011).

Equation (2) reflects that the local driving force on the front depends not only on the distance of the crack line to the reference position, but also on the full crack front configuration through the integral term embedding the long-range

1 The principal value PV $\int$ in Eq. (2) ensures the convergence of the integral. 

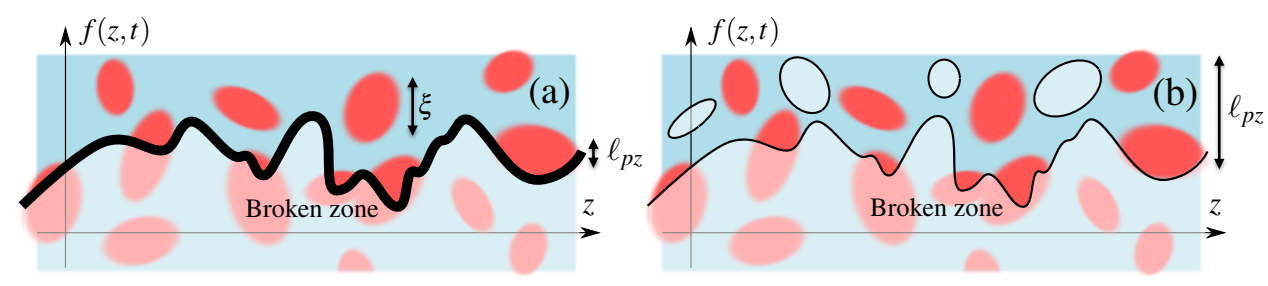

Fig. 4 Brittle vs quasi-brittle failure of heterogeneous materials. (a) For brittle failure, the process zone size $\ell_{p z}$ is much smaller than the characteristic microstructural length scale $\xi$ of the material. The crack growth process is well described by the motion of a sharp interface $f(z, t)$ separating the broken from the unbroken domain of the fracture plane. (b) For quasi-brittle failure, $\ell_{p z} \gg \xi$ so crack propagation is dominated by the processes of damage nucleation, growth and percolation.

interaction kernel $\sim 1 / z^{2}$. It means that a geometrical perturbation localized along the front affects the crack driving force everywhere else, but with a strength that decays as the inverse of the square of the distance. Such a non-local behavior is reminiscent of the underlying perturbations of the elastic field in the bulk ahead of the crack front: A local geometrical perturbation of the crack front produces an extended perturbation of the elastic field ahead of the crack in the still intact region of the material that ultimately modifies the elastic energy release rate everywhere along the front. To capture the essence of this non-local behavior, it is helpful to consider the Fourier transform of the integral term in Eq. (2) that follows $-|k| \delta \tilde{f}(k)$ where $\tilde{f}(k)$ is the Fourier transform of the front perturbations. Under this form, the elastic restoring force that applies on the front appears to be inversely proportional to the perturbation wavelength $1 / k$ so the smaller the wavelength, the stiffer the crack front.

The derivation of an evolution equation for the crack front builds on the perturbation of the Griffith's equilibrium condition $G[f(z, t)]=G_{\mathrm{c}}[f(z, t), \dot{f}(z, t)]$ that should be satisfied everywhere along the front at any time. Note that the variations of the fracture energy with the crack speed $\dot{f}=$ $\frac{\partial f}{\partial t}$ are also taken into account. We then follow the approach described previously where the elastic energy release rate has been expanded to the linear order in the crack front perturbation, and develop the fracture energy distribution along the front as

$\frac{G_{\mathrm{c}}(z, t)}{\overline{G_{\mathrm{c}}}}=1+\delta g_{\mathrm{c}}(z, x=f(z, t))+\frac{\dot{f}(z, t)-v_{\mathrm{m}}}{v_{0}}$.

The first term in this development describes the normalized toughness variations $g_{\mathrm{c}}(z, x)=\frac{G_{\mathrm{c}}\left(z, x, v_{m}\right)-\overline{G_{\mathrm{c}}}}{\overline{G_{\mathrm{c}}}}$ from a reference value $\overline{G_{\mathrm{c}}}=\left\langle G_{\mathrm{c}}\left(z, x, v_{\mathrm{m}}\right)\right\rangle_{z, x}$ defined as the average toughness within the fracture plane for the crack speed $v_{\mathrm{m}}=\langle\dot{f}(z, t)\rangle_{z, t}$. Note that we do not linearized this term along $f(z, t)-\langle f(z, t)\rangle_{z, t}$, as it would limit our calculation to small front perturbations with respect to the heterogeneity size $\xi$. The second term in Eq. (3) takes into account the variations of fracture energy that generally increases with crack speed. This variation is approximated by a linear relation with coefficient $G_{\mathrm{c}}^{\prime}=d\left\langle G_{\mathrm{c}}(x, z, \dot{f})\right\rangle_{z, x} /\left.d \dot{f}\right|_{\nu_{\mathrm{m}}}$. For practical purposes, we introduce the velocity $v_{0}=\overline{G_{\mathrm{c}}} / G_{\mathrm{c}}^{\prime}$ that is a characteristic constant of the material that may depend on the average crack speed $v_{\mathrm{m}}$.

The insertion of Eqs. (2) and (3) into the Griffith's equilibrium condition $G(z, t)=G_{\mathrm{c}}(z, t)$ gives the following crack evolution equation

$$
\frac{\dot{f}-v_{\mathrm{m}}}{v_{0}}=\frac{f_{0}-f}{\mathscr{L}}+\frac{1}{\pi} P V \int_{-\infty}^{+\infty} \frac{f(\tilde{z}, t)-f(z, t)}{(\tilde{z}-z)^{2}} d \tilde{z}-\delta g_{\mathrm{c}}(z, f) .
$$

It relies on the assumption of small toughness variations $\sqrt{\left\langle\delta g_{\mathrm{c}}(z, x)^{2}\right\rangle_{z, x}}<1$ that justifies the linear development of $G$ with the front perturbation in Eq. (2). It also assumes linear variations of $G_{0}(f)-G_{0}\left(f_{0}\right)$ and $G_{\mathrm{c}}(\dot{f})-G_{\mathrm{c}}\left(v_{\mathrm{m}}\right)$ within the range of crack length and speed investigated.

This equation of motion captures many features of the statistics of crack growth observed experimentally. In particular, it describes accurately the mechanism of collective pinning of the front by an assembly of obstacles at the origin of the giant fluctuations evidenced in Fig. 1(b). As a result, it has been used to interpret several experimental observations like the power law distribution of failure bursts measured at the local scale from spatio-temporal diagrams like the one of Figs. 2(a) and 3(a) (Bonamy et al., 2008), the scale invariant roughness of the front characterized by a roughness exponent $\zeta \simeq 0.4$ (Santucci et al., 2010) or the fine temporal structure of global avalanches and their asymmetry (Laurson et al., 2010). Here, we would like to focus on the distribution $P(v)$ as predicted from this equation that can help us to interpret the experimental results presented in Section 2.1.

The equation of motion (4) can be solved numericaly for values of the parameters $\mathscr{L}$ and $v_{0}$ in agreement with the experimental conditions. The predicted distribution of local velocity is shown in Fig. 5. Similarly to the experimental observations, it also shows two different regimes with a pinning regime at $v<\langle v\rangle$ corresponding to pinned crack configurations characterized by a power law with exponent $\eta_{p} \simeq 1.6$ and a depinning regime for $v>\langle v\rangle$ characterized 
by $\eta_{d} \simeq 2.0$. Note that the slopes of the distribution represented in Fig. 5 are actually $\eta_{d}-1$ and $\eta_{p}-1$, since the velocities have been defined here from the waiting times of the crack front following the procedure proposed by Tallakstad et al. (2011). The correspondance between the theoretically predicted and the experimentally measured exponents of Figs. 2(c) and 3(c) is not clear.

To disentangle the scaling behavior of the velocity distribution, it is useful to explore the dynamics of the crack in a simpler situation. Consider the relaxation of crack front as it recovers a straight configuration after depinning from a single obstacle. This problem can be addressed by considering the motion Equation (4) but with a homogeneous toughness field $g_{\mathrm{c}}=0$. The initial front geometry at the onset of depinning corresponds to the solution

$f(z)=\frac{C d}{\pi}\left[\left(1+\frac{z}{d}\right) \ln \left|1+\frac{z}{d}\right|+\left(1-\frac{z}{d}\right) \ln \left|1-\frac{z}{d}\right|\right]$

of the classical problem of a front at equilibrium in a fracture plane containing an isolated obstacle of width $2 d$ and toughness contrast $C=\left(G_{\mathrm{c}}^{O}-\overline{G_{\mathrm{c}}}\right) / \overline{G_{\mathrm{c}}}$ where $G_{\mathrm{c}}^{O}>\overline{G_{\mathrm{c}}}$ is the toughness of the obstacle (Chopin et al., 2011; Vasoya et al., 2013). The resolution of the relaxation dynamics provides the velocity field

$\dot{f}(z, t)=C v_{0}\left[1-\frac{1}{\pi}\left(\arctan \left(\frac{v_{0} t}{d+z}\right)+\arctan \left(\frac{v_{0} t}{d-z}\right)\right)\right] \sim$

for small defects $d \ll \mathscr{L}$ compared to the structural length introduced in Eq. (2). First, it provides a simple physical interpretation of the characteristic velocity $v_{0}$ introduced in the crack front evolution equation since it sets the initial velocity $\dot{f}(z, 0)=C v_{0}$ at the onset of depinning. Second, it predicts a front relaxation in $\dot{f} \sim 1 / t$ after a short transient $t \gg d / v_{0}$. One deduces from it the scaling behavior of the velocity distribution $P(v) \sim 1 / v^{2}$ during the micro-instability produced by the depinning of the front from a single obstacle.

Avalanches observed during the propagation of a crack through a disordered interfaces result from the depinning from several obstacles. However, our numerical resolutions show that the scaling of the velocity distribution remains similar and follows $P(v) \sim 1 / v^{2}$ irrespective of the avalanche size and so the number of obstacles involved in the depinning process.

An interpretation of the scaling behavior of the local velocity distribution is now in order. In the direct simulations of the crack growth equation, the power law statistics $P(v) \sim$ $1 / v^{2}$ observed in the depinning regime is the signature of the relaxation mechanisms when the front detaches from obstacles. This is consistent with the observation of a similar scaling in the experiments of Fig. 3 where a crack propagates at the disordered interface between a PDMS block and a rigid substrate. Indeed, for that particular experimental setup, the process zone size is of the order of a few tenth of nanometers (Ciccotti and Creton, 2015), much lower than the patterned heterogeneities introduced at the micrometer scale, guaranteeing that the hypothesis of brittle crack growth illustrated in Fig. 4 is satisfied.

The agreement between the LEFM based model and the experiments of Fig. 3 raises the question of the origin of the large exponent $\eta_{d} \simeq 2.5$ characterizing the depinning regime in the experiments on PMMA presented in Fig. 2. This deviation to the LEFM prediction can be understood by investigating the local crack front dynamics using a discrete model of fracture that goes beyond brittle fracture. Gjerden et al. (2014) investigated the propagation of a crack through a weak disordered interface separating two blocks connected by an array of parallel brittle fibers. When the force applied to one of the fiber exceeds its failure threshold, the fiber breaks and tensile forces are redistributed through the intact region of the interface assuming that blocks behave elastically. This redistribution mechanism produces cascades of failure events, qualitatively similar to the avalanche dynamics described by the LEFM based model of Eq. (4). For a weakly disordered interface, the simulation even recovers quantitatively the predictions of the depinning models and in particular the value of the roughness exponent $\zeta \simeq 0.4$ predicted from the LEFM. But a more interesting regime $\sim 1$ takes place for strongly disordered interfaces. Indeed, in that regime, the front dynamics is not governed by the competition between the elasticity of the crack line and the disorder, but instead by the coalescence of regions of broken fiber located ahead of the crack with the advancing crack itself. Interestingly, this transition from brittle to quasi-brittle crack growth reflects also on the velocity probability density: When the process of damage coalescence dominates, as illustrated in Fig. 4(b), the velocity distribution follows another scaling behavior $P(v) \simeq v^{-\eta_{\mathrm{d}}}$ with $\eta_{\mathrm{d}} \simeq 2.5$ as shown in Fig. 5(b). This finding is in excellent agreement with Måløy et al. (1992)'s experimental observations shown in Fig. 2(c).

The comparison between coalescence model and experiments suggests that crack growth between two sintered PMMA plates as performed in Måløy et al. (1992)'s experiments is dominated by damage coalescence. This point would certainly deserve further experimental investigations.

The existence of two distinct scaling regimes with exponent $\eta_{\mathrm{d}} \simeq 2.0$ for brittle failure and $\eta_{\mathrm{d}} \simeq 2.5$ for quasi-brittle crack growth also invites a discussion of Barés et al. (2013)'s experimental results presented in Fig. 1(c). Here, a scaling law with $\eta_{\mathrm{d}} \simeq 2.5$ was reported in the depinning regime. Since such scaling actually does survive to upscaling (Tallakstad et al., 2011), it is tempting to interpret this observation in terms of microscopic failure mechanism, and conjecture that microcracking does take place at a scale comparable to the grain size $\xi \simeq 500 \mu \mathrm{m}$ of the sintered materials used in these experiments. 

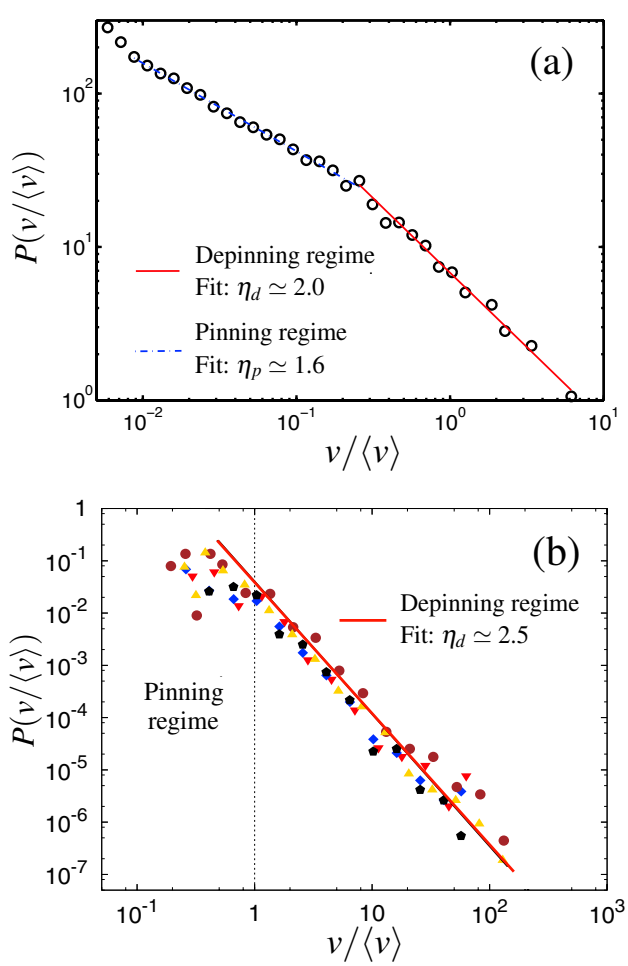

Fig. 5 Distribution of local crack speeds as predicted from (a) a LEFM based model that describes brittle crack propapation in a disordered material (see Eq. (4)); (b) a fiber bundle model that describes crack propagation as a damage percolation process [Courtesy of Gjerden et al. (2014)]. In both cases, the velocity distribution shows two regimes: The low velocity or pinning regime is reminiscent of zones of the front that are trapped by material heterogeneities while the large velocity or depinning regime describes the crack dynamics within avalanches. Note that the exponents involved in the depinning regimes are different in both models and capture well the difference also observed in the experiments (see Fig. 2(c) and 3(c)).

Many questions remain open. First, we have mainly focused on the depinning regime, and proposed an interpretation for the scaling behavior of the velocity distribution in terms of local crack growth mechanism. What about the pinning regime? The observation of a scaling behavior with an exponent $\eta_{\mathrm{p}} \simeq 1.4$ in Barés et al. (2013)'s experiment, close to the LEFM prediction $\eta_{\mathrm{p}} \simeq 1.6$ shown in Fig. 5(a), is indication that the velocity distribution in the pinning regime might be robust through the transition from brittle to quasibrittle crack growth.

To conclude, we illustrated in this part how the statistics of crack growth fluctuations observed either at the local scale or at the global one can be used as a probe to investigate the nature of microscale failure mechanisms. This opens interesting perspectives for the monitoring of structures from a statistical treatment of acoustic signals emitted during their progressive fracture and damage. We now move to the study of fracture surfaces statistics that also provide
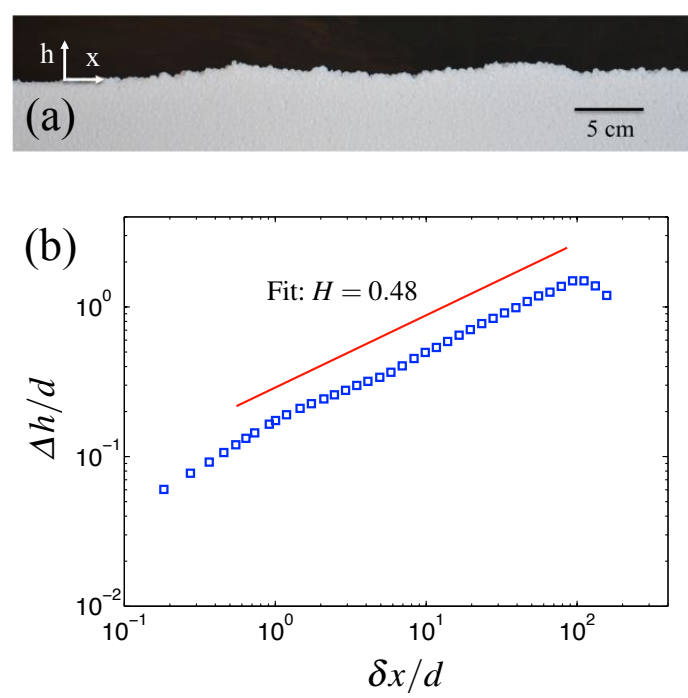

Fig. 6 (a) Fracture profile in a thin panel of expanded polystyrene made of beads of size $d \simeq 2 \mathrm{~mm}$. (b) Logarithmic representation of the height-height correlation fonction of the fracture profiles. At scales larger than the bead size $d$, crack roughness is self-affine with an exponent $H=0.48 \pm 0.05$.

a rich information on elementary mechanisms involved in crack growth.

\section{Statistics of fluctuations in the trajectory of cracks}

Fracture surfaces can be considered as the Holy Grail for models of crack propagation in disordered materials, as they have been extensively used as a benchmark to compare and discriminate competing approaches (Bouchaud et al., 1993; Hansen and Schmittbuhl, 2003; Ponson et al., 2006c; Nukala et al., 2010). Here, we would like to illustrate how to take advantage of the dialogue between theory and experiment to identify basic crack growth mechanisms from the statistics of fracture surfaces. And propose fracture surface geometry as a paradigm for understanding failure phenomena in disordered materials.

\subsection{Crack path in thin sheets}

To isolate the basic mechanisms underlying crack path selection in heterogeneous media, it is instructive to consider first crack growth in 2D thin sheets. By thin sheets, we mean structures with a small thickness compared to their width and length, but also smaller than or comparable to the characteristic microstructural feature of the material.

Figures 6(a) and 7(a) show fracture profiles in a panel of expanded polystyrene made of $d \simeq 2 \mathrm{~mm}$ size beads and in a paper sheet, respectively. After digitizing the crack paths 

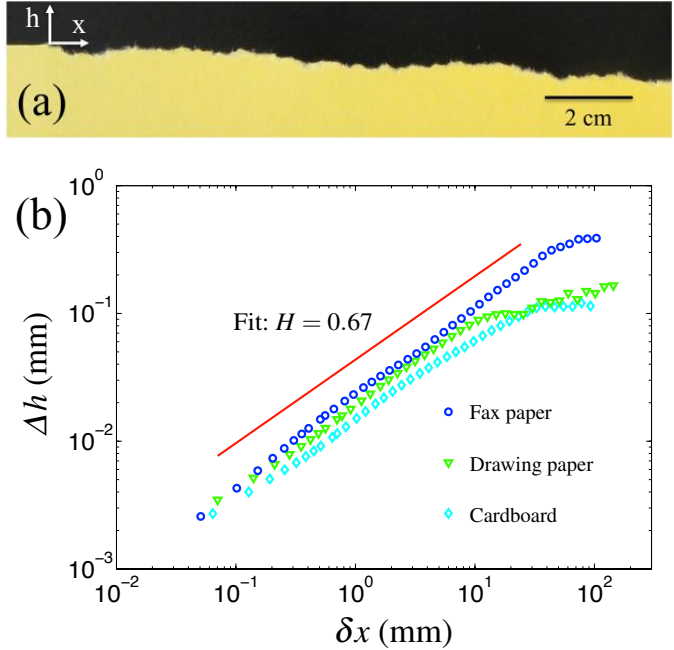

Fig. 7 (a) Fracture profile in a sheet of drawing paper. (b) Logarithmic representation of the height-height correlation of fracture profiles in three different types of paper. The fracture roughness shows a selfaffine behavior characterized by an exponent $H=0.67 \pm 0.05$ that varies weakly from one type of paper to another.

$h(x)$, their geometry can be characterized through their heightheight correlation function

$\Delta h(\delta x)=\left\langle[h(x+\delta x)-h(x)]^{2}\right\rangle_{x}^{1 / 2}$.

where $\Delta h$ is the height difference between two points along the crack path $h(x)$ separated by the distance $\delta x$ along the average crack line $x . \Delta h$ is also averaged over several samples of the same material broken under the same loading conditions to obtain a smooth variation with $\delta x$. Figures 6(b) and 7(b) show the crack correlation functions thus obtained for three types of paper sheet and for the polystyrene panel. They both follow a power law $\Delta h \simeq \delta x^{H}$ which is reminiscent of self-affine properties. However, the value of the characteristic exponent, also referred to as the Hurst exponent, differs significantly with $H=0.48 \pm 0.05$ in polystyrene panels and $H=0.67 \pm 0.05$ in paper sheets.

This finding indicates two dramatically different fracture behaviors. Cracks in poystyrene panels with $H \simeq 1 / 2$ follow trajectories close to a directed random walk: At any time during failure, the crack has the same probability to propagate upward as downward, irrespective of the prior propagation direction. As clear from Fig. 6(b) where the axes have been normalized by the bead size, the random walk behavior starts at a scale $\delta x \simeq d$ up to a cutoff length $\delta x \simeq 100 d$. Since the self-affine crack geometry reflects the random microstructure of the material, the elementary microstructural feature sets the lower bound of the scale invariant regime. The upper bound has a different origin: It emerges from the finite size of the specimen, as shown by studying the effect of the sample dimensions on the fracture surface scaling properties (Ponson et al., 2007).
For fractures in paper sheets that display exponents $H \simeq$ 0.7 larger than $1 / 2$, trajectories are persistent random walks. Crack deflections towards the upper $h^{\prime}(x)>0$ (resp. lower $\left.h^{\prime}(x)<0\right)$ direction will be more likely followed by a positive (resp. negative) subsequent deflection. The lower bound of the self-affine regime is less clear for paper sheets than for polystyrene panels, and we will explain this observation later. The upper bound however, as for polystyrene panels, can be shown to emerge from the finite size of the specimen.

To make sense to these observations, the geometry of cracks in brittle materials with disordered fracture properties is explored theoretically. The model proposed here relies on the assumption that the crack follows the direction in which the shear component of the loading cancels out (Gol'dstein and Salganik, 1974). Using this so-called principle of local symmetry actually amounts to assume that the process zone size $\ell_{\mathrm{pz}}$ is much smaller than the size of the microstructural features at the origin of the crack deflection, so that LEFM can be safely applied. We will see that this hypothesis plays a central role in the interpretation of the two roughening behaviors observed experimentally. From the principle of local symmetry and the expression of the local stress intensity factors in tension $k_{I}\{h(x)\}$ and shear $k_{I I}\{h(x)\}$ for slightly perturbed crack trajectory, one derives the following path equation

$\frac{\mathrm{d} h}{\mathrm{~d} x}=-\frac{1}{\sqrt{\mathscr{L}_{1}}} \int_{-\infty}^{x} \frac{h^{\prime}(\tilde{x})}{\sqrt{x-\tilde{x}}} \mathrm{~d} \tilde{x}-\frac{h(x)}{\mathscr{L}_{2}}+\eta(x)$

that is valid in the limit of small crack deflections $h^{\prime}(x) \ll 1$. Note that contrary to Katzav et al. (2007), the material elastic heterogeneities are not taken into account, resulting in a simplified version of the path equation that they derived. The term $\eta(x)$ is a quenched noise that describes the effect of the toughness variations and anisotropy resulting from the disordered material microstructure. Its value change randomly over $-\eta_{0} \leq \eta \leq \eta_{0}$ each time the crack propagates over a distance of the order of the characteritic microstructural size. The lengths $\mathscr{L}_{1} \simeq\left(T / K_{\mathrm{I}}\right)^{2}$ and $\mathscr{L}_{2} \simeq A_{\mathrm{I}} / K_{\mathrm{I}}$ involved in the path equation (8) relates to the values of the coefficients $K_{\mathrm{I}}$, $T$ and $A_{\mathrm{I}}$ in the Williams' development of the stress field in the tip vicinity of the unperturbed straight crack. In particular, the T-stress, negative in the experiments described here, controls the stability of the crack trajectory (Cotterell and Rice, 1980). The calculation of these lengths for the actual fracture tests shown in Fig. 6(a) and 7(b) gives $\mathscr{L}_{1} \simeq \mathscr{L}_{2} \simeq$ $100 \mathrm{~d}$ for the polystyrene panels and $\mathscr{L}_{1} \simeq \mathscr{L}_{2} \simeq 10 \mathrm{~cm}$ for the paper sheets: They are of the order of the specimen in-plane dimension and much larger than the characteristic size of the microstructural features. Under these conditions, the first two terms in the path equation that scale as $\sim 1 / \sqrt{\mathscr{L}_{1}}$ and $\sim 1 / \mathscr{L}_{2}$ become negligible. The approximated path equation $\mathrm{d} h / \mathrm{d} x \simeq \eta(x)$ thus obtained is char- 
acteristic of a directed random walk. It predicts self-affine crack profiles with an exponent $H=1 / 2$.

This theoretical analysis of the crack paths in 2D disordered brittle solids captures the observations made in Fig. 6 for polystyrene panels, namely uncorrelated crack deflections reflected by the random walk exponent $H \simeq 0.5$. This agreement lies in the peculiar failure mechanism of expanded polystyrene: As the crack meanders through its poorly consolidated granular structure, the actual dissipative failure mechanisms taking place in the crack tip vicinity are confined in a process zone of size $\ell_{\mathrm{pz}}$ much smaller than the size $d$ of the polystyrene beads. Therefore, the crack deflection mechanism in this material is fairly well described under the assumption of a brittle crack growth as supposed in the model.

The behavior of cracks in paper sheets with $H>1 / 2$ indicate another roughening mechanism. Here, the characteristic scale of the microstructure, namely fibers of length $d \simeq 0.1-1 \mathrm{~mm}$ (Ververis et al., 2004), compares with the characteristic scale of damage processes. A closer look at the failure mechanisms in paper indeed reveal that macroscopic cracks propagate through the nucleation and growth of mm scale microcracks that subsequently coalesce with it. This discontinuous growth process results in jumps of the crack tip from one position to another that can be evidenced using a fast camera or through the acoustic bursts emitted during failure (Stojanova et al., 2013).

These local failure mechanisms reflect on the crack roughness at the large scale. As shown by Ben-Dayan et al. (2006), crack propagation by damage nucleation and coalescence produces persistent self-affine crack trajectories characterized by a Hurst exponent larger than $1 / 2$, equal to $H \simeq$ 0.65 in their specific model. Roughly speaking, the positive correlations that build along the crack path emerges from the attraction exerted by the microcrack on the main crack through the following mechanism: As the level of tensile stress is maximum along the current crack propagation direction, microcracks are likely to nucleate in this direction. Once damage nucleation takes place, microcrack and crack attract each other, so the main crack is now more likely to propagate in its current propagation direction. Such a roughening mechanism was also observed through simulations of crack propagation by damage growth and coalescence in ductile materials (Srivastava et al., 2014). The roughness exponent $H \simeq 0.55$ characterizing the fracture surfaces in their simulations is lower than the one found by Ben-Dayan et al. (2006), however significantly larger than $1 / 2$ to indicate persistency in the crack trajectory.

The link between failure mechanisms and roughness properties in thin sheets is clear from the experiments and the models: Brittle crack growth in the limit of large specimens leads to uncorrelated random fracture profiles $(H \simeq 0.5)$ while crack propagation through damage nucleation results in positive correlations between successive crack growth incre- ments $(H>1 / 2)$. The comparison between two length scales, namely the characteristic size $d$ of the elementary microstructural feature and the characteristic size $\ell_{\mathrm{pz}}$ of the damage processes, is proposed as a criterion to distinguish both mechanisms. We now move to the study of the fracture surfaces of fully three-dimensional materials that does reveal the competing roughening mechanisms evidenced in thin sheets, but involves a different selection process between them.

\subsection{Roughness of two-dimensional fracture surfaces}

We now consider fracture in specimens with a large dimension along the crack front direction compared to the characteristic microstructural size of the material. In that situation, the crack leaves behind it a 2D fracture map as the ones shown in Fig. 8. Three materials are considered, namely an aluminum alloy, a mortar and a ceramic, with a priori three different failure behaviors to elicit roughness properties common to a large range of materials. The topography $h(z, x)$ of the fracture surface of each of these materials is measured through an adapted profilometric technique as detailed by Pouchou et al. (2002), Morel et al. (2008) and Ponson et al. (2006a), respectively.

The standard approach to characterize the geometry of fracture surfaces is to compute their height-height correlation function, as defined in Eq. (7), either along the propagation direction $x$ or the perpendicular one $z$. A more complete characterization consists in computing their $2 \mathrm{D}$ correlation function $\Delta h(\delta \mathbf{x})=\left\langle[h(\mathbf{x}+\delta \mathbf{x})-h(\mathbf{x})]^{2}\right\rangle_{\mathbf{x}}^{1 / 2}$ that allows for the description of their anistropic scaling properties (Ponson et al., 2006b). Previous works have shown that fracture surfaces are self-affine, namely that their correlation function follows a power law $\Delta h \sim \delta z^{\zeta}$ with an exponent $\zeta \simeq 0.75$ that was conjectured to be universal (Bouchaud et al., 1990; Måløy et al., 1992). However, more recently, another roughness behavior was reported on brittle rocks with a lower roughness exponent $\zeta \simeq 0.45$ (Boffa et al., 1998; Ponson et al., 2006a). Following the work of Santucci et al. (2007), we would like here to take a step back from the scaling properties of fracture surfaces and investigate the underlying statistics of height fluctuations to reveal the range of length scales over which roughness shows meaningful correlations.

Figure 8 shows the distribution $P_{\delta r}$ of height variations $\delta h=h(\mathbf{x}+\delta \mathbf{x})-h(\mathbf{x})$ where the sampling is done on all admissible $\mathbf{x}$ and $\delta \mathbf{x}$ such as $\delta r=|\delta \mathbf{x}|$. In other words, we focus on the height variations at different scales $\delta r$ and treat fracture surfaces as isotropic maps. An interesting property of the family of distributions $P_{\delta r}$ is that they follow a Gaussian behavior at large length scales $\delta r \gg \xi$ while they exhibit fat tail statistics at small length scales $\delta r \ll \xi$ as evidenced from the comparison with parabolas characteristic of Gaussian distributions in the semi-logarithmic represen- 

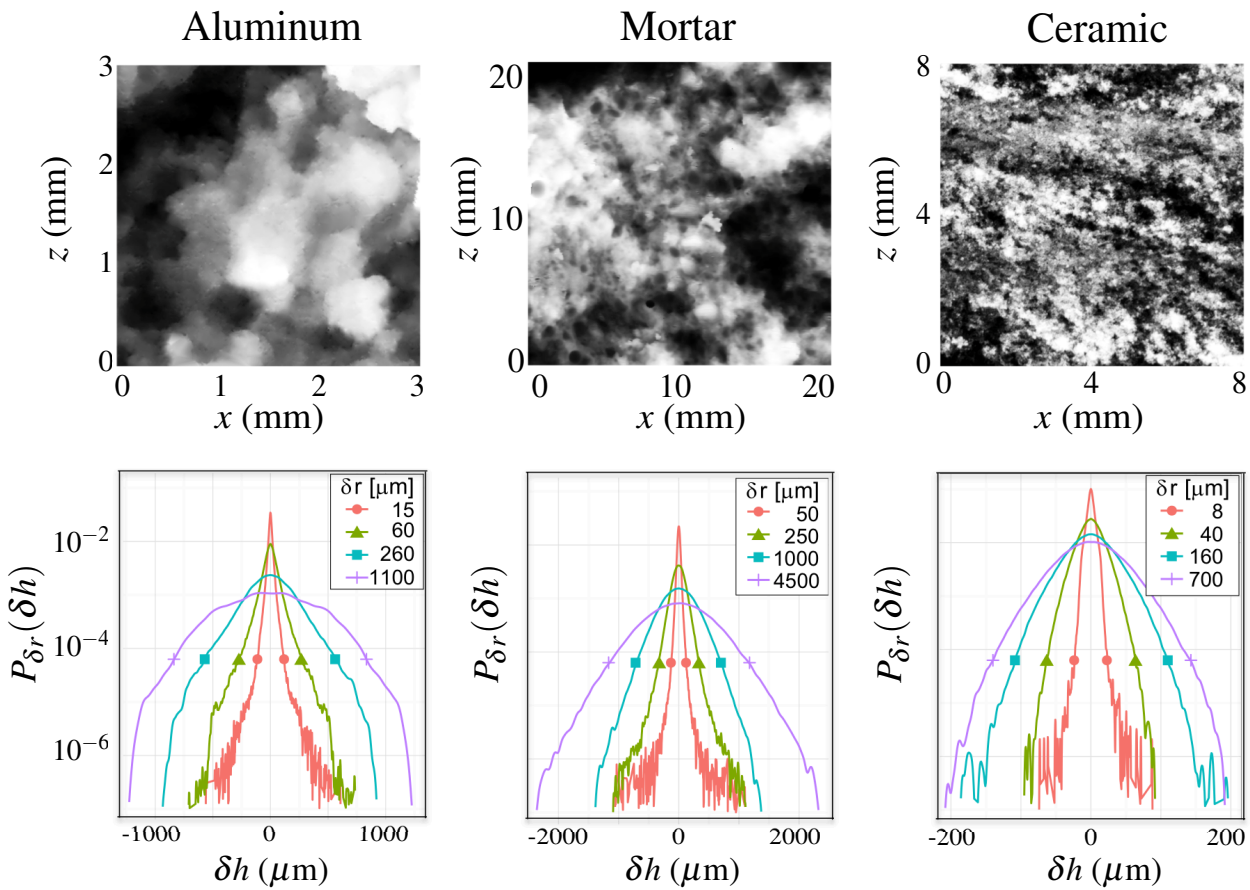

Fig. 8 Height maps $h(\mathbf{x})$ of fracture surfaces of aluminum, mortar and ceramic. Their distributions of height variations computed at different scales $\delta r=|\mathbf{x}|$ show a Gaussian behavior at large scales $\delta r \gg \xi$ against fat tail behavior at small scales $\delta r \ll \xi$ where $\xi$ is a material dependant length scale measured in Fig. 9. [Courtesy of Vernède et al. (2015)]

tation of Fig. 8. To measure the crossover length scale $\xi$ between fat tail and Gaussian statistics, we introduce the following operator

$\omega(\mathbf{x})=\frac{1}{2} \log \left(\left\langle\delta h(\mathbf{x}, \delta \mathbf{x})^{2}\right\rangle_{|\delta \mathbf{x}|=\varepsilon}\right)-\Omega_{\varepsilon}$.

It transforms the original height map $h(\mathbf{x})$ into a map $\omega(\mathbf{x})$ of the local roughness level that is defined from an average of the height variations over a circle of radius $\varepsilon$ centered in $\mathbf{x} .^{2}$ The fields $\omega$ computed for the three fracture surfaces considered are shown in Fig. 9. The patterns that emerge on the fracture surface through this transformation correspond to steep cliffs and reveal complex correlations of the height variations. Their characteristic size is reminiscent of the length scale $\xi$ evidenced from the variations of $P_{\delta r}$ with $\delta r$. Indeed, first write the height variation computed at a scale $\delta r$ as the sum of several height variations computed at a finer scale $\varepsilon \ll \delta r,{ }^{3}$ and then use the central limit theorem: If the fluctuations at the scale $\varepsilon$ are uncorrelated, then $P_{\delta r \gg \varepsilon}$ is a Gaussian. Conversely, if the distribution $P_{\delta r}$ is non-Gaussian, this indicates spatial correlations of height

\footnotetext{
2 The constant $\Omega_{\varepsilon}$ involved in Eq. (9) is chosen such that the average of $\omega(\mathbf{x})$ over all $\mathbf{x}$ is zero.

3 The actual decomposition of the height variation computed as a scale $\delta r$ into the sum of heigth variations computed at a finer scale $\varepsilon=$ $\delta r / n$ where $n$ is an integer writes as $\delta h(\mathbf{x}, \delta \mathbf{x})=h(\mathbf{x}+\delta \mathbf{x})-h(\mathbf{x})=$ $\sum_{k=1}^{n} h\left(\mathbf{x}+\frac{k}{n} \delta \mathbf{x}\right)-h\left(\mathbf{x}+\frac{k-1}{n} \delta \mathbf{x}\right)=\sum_{k=1}^{n} \delta h\left(\mathbf{x}+\frac{k-1}{n} \delta \mathbf{x}, \frac{\delta \mathbf{x}}{n}\right)$
}

variations at a scale $\delta r$ or smaller. As a result, the crossover length identified from the transition from fat tail to Gaussian statistics does correspond to the correlation length of the $\omega$ maps, and we note both length scales $\xi$ in the following.

The correlations of $\omega$ are studied through the function $C(\delta r)=\langle\omega(\mathbf{x}) \omega(\mathbf{x}+\delta \mathbf{x})\rangle_{\mathbf{x},|\delta \mathbf{x}|=\delta r}$ shown in Fig. 9 as a function of the distance $\delta r$ for different values of $\varepsilon ; C(\delta r)$ is in fact independent of $\varepsilon$ whenever $\varepsilon \ll \delta r$. For the three materials considered, we observe two regimes: At small $\delta r$, $\omega$ shows strong spatial correlations which decay logarithmically as $C(\delta r) \sim-\log (\delta r / \xi)$ and extrapolates to zero for $\delta r=\xi$. For larger distances, these correlations are zero within statistical noise. $\xi$ corresponds to the characteristic size of the patterns of the $\omega$-fields shown in Fig. 9.

The presence of two separate ranges of length scales with distinct statistical properties on the fracture surfaces of these materials is clear: At small $\delta r \ll \xi$, the height fluctuations are strongly correlated and display non-Gaussian statistics while at large $\delta r \gg \xi$, the roughness follows a Gaussian behavior with no spatial correlation of the $\omega$-field. We would like now to come back on the self-affine properties of the fracture surface and determine the value of the roughness exponent in these both regimes.

Figure 10 shows the correlation function $\Delta h(\delta r)$ of the three fracture surfaces following the definition of Eq. (7) after averaging over all the possible directions such that $\delta r=$ $|\delta \mathbf{x}|$. The two ranges of length scales determined previously 

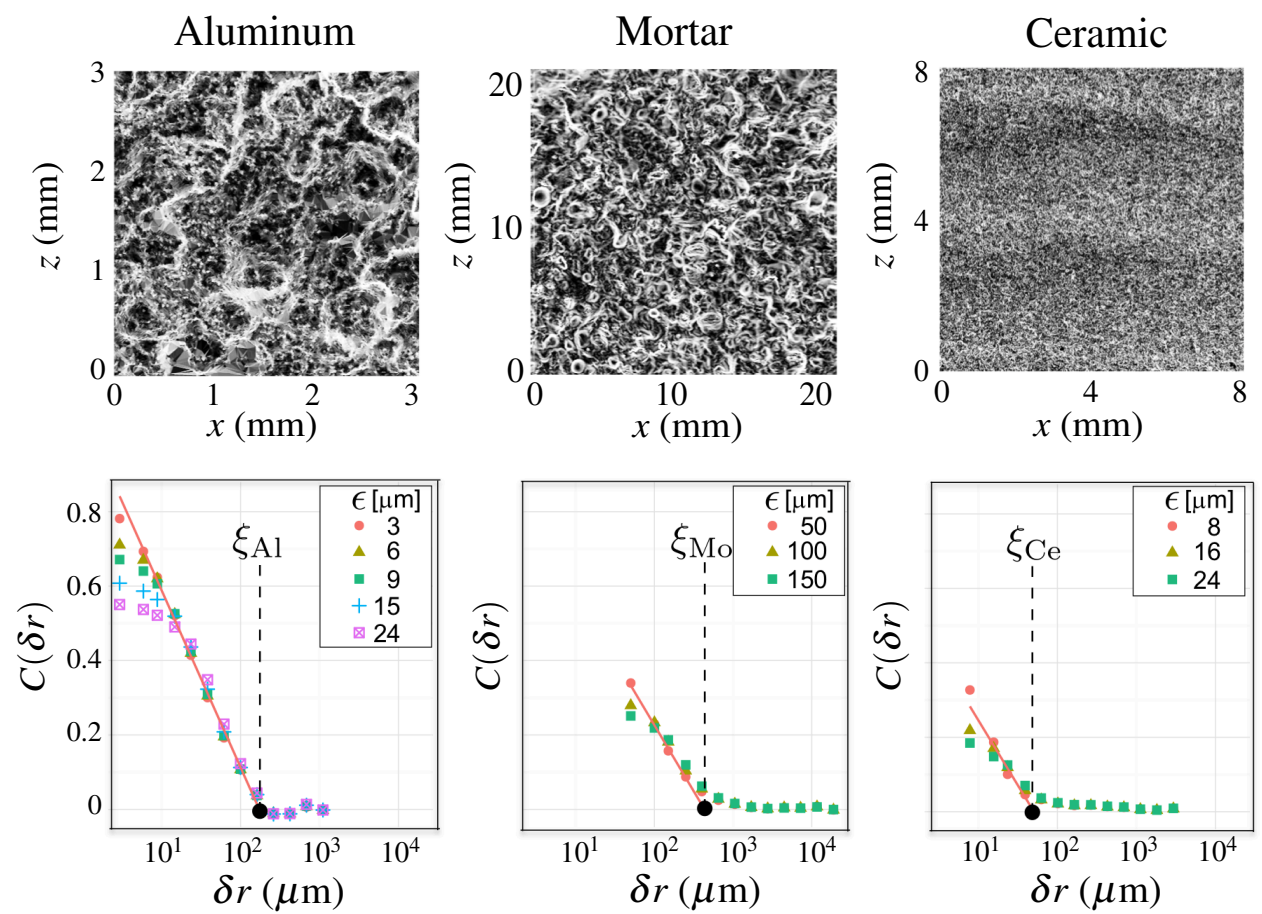

Fig. 9 Fracture surfaces shown in Fig. 8 processed through Eq. (9) to highlight the steepest cliffs that are shown in white. The characteristic size $\xi$ of the patterns visible on these maps is obtained from the variations of their correlation function $C(\delta r) \sim \log (\delta r / \xi)$ and gives $\xi=170 \mu$ m, $430 \mu \mathrm{m}$ and $170 \mu \mathrm{m}$ for the aluminum, the mortar and the ceramics fracture surface, respectively. [Courtesy of Vernède et al. (2015)]
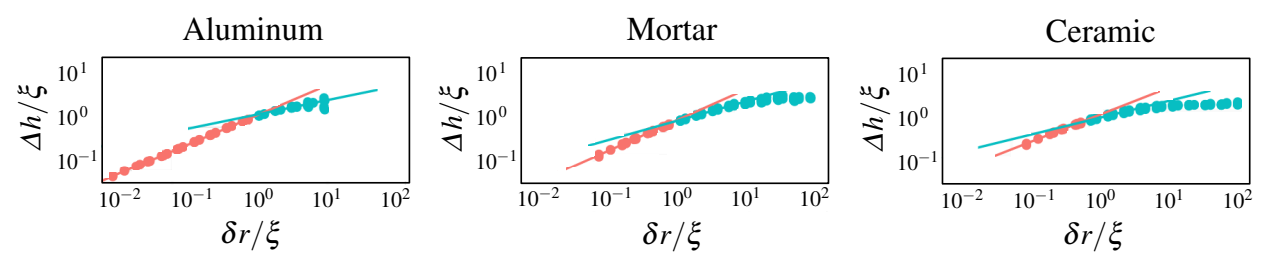

Fig. 10 Correlation function of the three fracture surfaces considered. The power law fits are performed below and above the length $\xi$ determined in Fig. 9 and give $\zeta=0.74 \pm 0.04$ at small scale and $\zeta=0.42 \pm 0.07$ at large scale. [Courtesy of Vernède et al. (2015)]

are indicated by two different colors. They do show two different scaling behaviors: At small $\delta r<\xi$, the correlation function follows $\Delta h \sim \delta r^{\zeta}$ with a roughness exponent $\zeta \simeq 0.75 \pm 0.05$, irrespective of the material considered. At larger $\delta r>\xi$, the roughness is characterized by a lower exponent $\zeta=0.45 \pm 0.05$ here also robust and independent on the material. As a result, the presence of two regimes evidenced in the statistics of height fluctuations is also clear from the self-affine properties of the height fracture maps that show a persistent behavior $\zeta>1 / 2$ at small $\delta r<\xi$ and an anti-persistent one $\zeta<1 / 2$ at large $\delta r>\xi$. The following section addresses the physical origin of these two regimes.

\subsection{Roughness statistics as a paradigm for crack growth phenomena?}

The analysis of Section 3.1 of the fracture profiles in 2D thin specimens gives indication on the mechanisms underlying the roughness properties observed for $3 \mathrm{D}$ solids. In $2 \mathrm{D}$ solids, persistency $(H>1 / 2)$ of fracture profiles is reminiscent of crack growth governed by damage coalescence processes while pure random walk behavior $H=1 / 2$ results from brittle fracture. Theoretical analyses of crack propagation in brittle media reveals that the major difference of 3D situations compared to 2D lies in the effective elasticity of the crack line that opposes to out-of-plane crack excursions (Larralde and Ball, 1995; Ramanathan et al., 1997; Movchan et al., 1998). This effect was argued to explain the anti-persistent roughness $(\zeta \simeq 0.45)$ reported in porous brittle rocks (Ponson et al., 2006a; Bonamy et al., 2006a) and the logarithmic $(\zeta=0)$ height correlations reported for 
phase-separated glasses (Dalmas et al., 2008). Therefore, the anti-persistent roughness regime $\zeta \simeq 0.45$ observed at large scale $\delta r>\xi$ on the three materials considered here is interpreted as the result of brittle crack growth. At these length scales, the material can indeed be identified as a coarsegrained equivalent linear elastic medium and LEFM can safely be applied.

A quantitative understanding of the small scale $\delta r<\xi$ roughness regime is still missing. However, Vernède et al. (2015) characterized the geometry of the patterns present on the $\omega$-maps of Fig. 9 and showed that they display remarkably robust features, like $e$.g. a fractal geometry with dimension $D=1.70 \pm 0.05$ irrespective of the material considered, suggesting a common underlying mechanism. Inspired by Ravi-Chandar and Yang (1997) and Guerra et al. (2012) who characterized the geometry of the marks left by microcracks on PMMA fracture surfaces, Vernède et al. (2015) conjectured that the steep cliffs evidenced in the $\omega$-maps of aluminum, mortar and ceramics are the footprints of damage coalescence. The particularity of PMMA compared to these three materials is that microcracking processes take place at a much larger scale than the microstructural features, leaving on fracture surfaces conic marks characteristic of the interaction between two microcracks in a homogeneous material. On the contrary, the interplay between material disorder and damage coalescence may result in the complex entangled lines evidenced in the $\omega$-fields of Fig. 9 for aluminum, mortar and ceramics.

What are the physical implications of the fracture surface properties identified in this study? At first, the universal statistical structure of crack roughness evidenced in this work recovers the traditional text-book picture of Fig. 11 of crack propagation phenomena: Dissipative failure mechanisms are confined at the crack tip vicinity in the so-called process zone while LEFM applies at larger distances $r \gg \ell_{\mathrm{pz}}$ from the crack tip where the material recovers a linear elastic behavior and the stress field the theoretical predicted $1 / \sqrt{r}$ singularity (Irwin, 1958). However, it goes beyond this classical description as the statistics of the fracture surfaces evidenced at small scales does display universal features indendendent of the material investigated. This is an important observation that suggests that, at small scales too, a common description of the crack growth process through damage coalescence does exist, and that this description does survive to the material specific dissipative mechanisms taking place within the process zone.

Do these observations find applications in fractography for the post-mortem characterization of material failure? From our observations, it is natural to interpret the length scale $\xi$ emerging from the fracture surface statistics as a measure of the fracture process zone size $\ell_{\mathrm{pz}}$. The observation made by Srivastava et al. (2014) of a linear relation between the length scale $\xi$ and the fracture toughness $G_{\mathrm{c}}$ in simulations

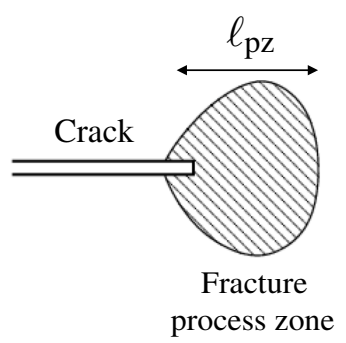

Fig. 11 Dissipative mechanisms during material failure are localized at the crack tip vicinity in a process zone of characteristic size $\ell_{\mathrm{pz}}$. The fracture surfaces of aluminum, mortar and ceramics reflects this phenomenon as they display two distinct statistical behaviors at small $\delta r<\xi$ and large $\delta r>\xi$ length scales, reminiscent of damage coalescence and brittle crack growth, respectively.

of ductile crack growth in heterogeneous solids does support this idea. Indeed, cohesive zone approach to fracture problems does predict a linear variation $G_{\mathrm{c}} \sim \ell_{\mathrm{pz}}$ of the toughness with the process zone size (Barenblatt, 1962). Further investigations of the effect of damage on fracture roughness are required. However, these observations are encouraging, and we are closer than ever to confirm the conjecture proposed 30 years ago by Mandelbrot et al. (1984) regarding a link between toughness and roughness. ${ }^{4}$ If it was confirmed, the statistical analysis of fracture surfaces could be an efficient way to measure material toughness at different scales and different locations in an already broken material. And also to estimate post-mortem the load applied to a material or a structure and help to determine the root cause of its failure.

\section{Conclusions and perspectives}

The statistical analysis of crack propagation in heterogeneous materials shows that the competition between material disorder and elasticity results in scale invariant fluctuations. This contrast with fracture patterns observed in homogeneous systems that often display a characteristic length scale like e.g. in columnar joints (Goehring et al., 2009) or in thin films (Marthelot et al., 2014). Therefore, the interpretation of the exponents characterizing crack growth fluctuations is key to make sense of these observations. In this article, we propose a connection between these scaling behaviors and two elementary mechanisms underlying material failure, namely brittle growth dominated by crack pinning and quasi-brittle growth dominated by damage coalescence. A lesson to be learnt from the statistics of fracture surfaces is that both mechanisms are generally present, but at different length scales.

\footnotetext{
4 Note however that the idea of Mandelbrot et al. (1984) was to establish a correlation of the material toughness with the roughness exponent, and not with a crossover length scale between two self-affine regimes as suggested by these recent studies.
} 
Another instructive finding is that the statistical properties of fluctuations emerging in the brittle regime are not more universal than the one observed in the damage regime. Since LEFM captures the main features of brittle failure for a large range of materials, this suggests that another complementary theoretical framework that is still to be developed can describe crack growth driven by damage coalescence.

What are the promising research directions along this line? Failure results from the coalescence of an assembly of microcracks growing in interaction. The related problem of damage spreading and localization prior to the emergence of a macroscopic crack has been studied within the frame of damage mechanics. Future theoretical developments should undoubtedly build on the basic concepts developed along these lines that can capture multiple cracks interaction (Kachano 2003) and localization phenomena (Pijaudier-Cabot and Bazant, 1987). But first, theoretical tools of damage mechanics must be adapted to heterogeneous media and include physically motivated criterion for damage nucleation that has been less developped. Then, these tools must be adapted to the specific loading conditions present at the vicinity of crack tip within the process zone.

The other challenge is experimental. Statistics in the dynamics of cracks and their trajectory have been a preferred direction of investigation due to the easy access to the information stored in the fracture surfaces and in the acoustic emission recorded during fracture. These indirect observations have been interpreted from statistical models of fracture following the approach described in this article. However, an easier way from the perspective of model validation and development, but more challenging from an experimental point of view, is to perform direct observations of failure mechanisms within the process zone. This requires adapted experimental techniques so that these in situ investigations can be performed at the proper length scale, e.g. the nanoscale in glass (Bonamy et al., 2006b) and the micronscale in polymers (Réthoré and Estevez, 2013). These observations limited to the free surface of the fracturing materials can be combined with digital image correlation techniques to measure the relevant mechanical fields (Hild et al., 2015; Han et al., 2010). Along the same line, the recent use of tomographic images (Limodin et al., 2009; Maire and Withers, 2014) for fracture problems already show promising results. An alternative approach is to scale up the dissipative failure processes by using model materials suitably designed for mimicking failure processes involved in traditional materials, but at a more appropriate length scale for in-situ observations. Additive manufacturing techniques might be an easy road for the fabrication of such systems (Dimas et al., 2013).

In the quest for increasingly smaller length and time scales of observation of failure processes, computational fracture mechanics has a central role to play. The development of numerical tools like molecular dynamics (Bitzek et al., 2015), discrete element models (Kun et al., 2014), and continuum mechanics-based simulations for brittle (Bourdin et al., 2000) and ductile failure (Needleman et al., 2012) allows for a realistic description of microscale failure processes. They have recently been helpful for interpreting the statistical properties of fluctuations in material failure and they are powerful means of investigation of the effect of microscale material features and processes on the failure behavior of solids.

Understanding the interplay between damage and disorder during crack growth is indeed the next step in the development of predictive models that bridge material microstructure to their failure behavior; and ultimately, that asovists the design of materials with improved failure properties - a major challenge indeed.

Acknowledgments The author would like to acknowledge his colleagues Daniel Bonamy, Jean-Philippe Bouchaud, Julien Chopin, Angelo Simone and Stephane Vernède for their contributions to this work and thank Elisabeth Bouchaud and Jean-Baptiste Leblond for their invaluable support.

\section{References}

Alava, M. J., P. K. Nukala, and S. Zapperi: 2006, 'Statistical models of fracture'. Adv. Phys. 55, 349-476.

Barenblatt, G. I.: 1962, 'The mathematical theory of equilibrium cracks in brittle fracture'. Adv. Appl. Mech. 7, 55-129.

Barés, J., L. Barbier, and D. Bonamy: 2013, 'Crackling versus continuumlike dynamics in brittle failure'. Phys. Rev. Lett. 111, 054301.

Barés, J., M. L. Hattali, D. Dalmas, and D. Bonamy: 2014, 'Fluctuations of global energy release and crackling in nominally brittle heterogeneous fracture'. Phys. Rev. Lett. 113, 264301.

Ben-Dayan, I., E. Bouchbinder, and I. Procaccia: 2006, 'Random and correlated roughening in slow fracture by damage nucleation'. Phys. Rev. E 74, 146102.

Bitzek, E., J. R. Kermode, and P. Gumbsch: 2015, 'Atomistic aspects of fracture'. Int. J. Frac. 191, 13-30.

Boffa, J. M., C. Allain, and J. P. Hulin: 1998, 'Experimental analysis of fracture rugosity in granular and compact rocks'. Eur. Phys. J. Appl. Phys. 2, 281-289.

Bonamy, D. and E. Bouchaud: 2011, 'Failure of heterogeneous materials: a dynamic phase transition?'. Phys. Rep. 498, 1-44.

Bonamy, D., L. Ponson, S. Prades, E. Bouchaud, and C. Guillot: 2006a, 'Scaling exponents for fracture surfaces in homogeneous glass and glassy ceramics'. Phys. Rev. Lett. 97, 135504.

Bonamy, D., S. Prades, C. L. Rountree, L. Ponson, D. Dalmas, E. Bouchaud, K. Ravi-Chandar, and C. Guillot: 
2006b, 'Nanoscale damage during fracture in silica glass'. Int. J. Frac. 140, 3-13.

Bonamy, D., S. Santucci, and L. Ponson: 2008, 'Crackling dynamics in material failure as the signature of a selforganized dynamic phase transition'. Phys. Rev. Lett. 101, 045501.

Bouchaud, E., G. Lapasset, and J. Planès: 1990, 'Fractal dimension of fractured surfaces: A universal value?'. Europhys. Lett. 13, 73-79.

Bouchaud, J. P., E. Bouchaud, G. Lapasset, and J. Planès: 1993, 'Models of fractal cracks'. Phys. Rev. Lett. 71, 2240-2243.

Bourdin, B., G. A. Francfort, and J. J. Marigo: 2000, 'Numerical experimnets in revisited brittle fracture'. J. Mech. Phys. Solids 48, 797 - 826.

Chopin, J., E. Bouchaud, and L. Ponson, 'Intermittent crack dynamics through disordered interfaces with controlled fracture properties'. (in preparation).

Chopin, J., A. Boudaoud, and M. Adda-Bedia: 2015, 'Morhology and dynamics of a crack front propagating in a model disordered material'. J. Mech. Phys. Solids 74, $38-48$.

Chopin, J., A. Prevost, A. Boudaoud, and M. Adda-Bedia: 2011, 'Crack front dynamics across a single heterogeneity’. Phys. Rev. Lett. 107, 144301.

Ciccotti, M. and C. Creton: 2015, 'Fracture and adhesion of soft materials: A review'. Prog. Poly. Sci. (in press).

Clotet, X., J. Ortin, and S. Santucci: 2014, 'DisorderInduced Capillary Bursts Control Intermittency in Slow Imbibition'. Phys. Rev. Lett. 113(074501).

Cotterell, B. and J. R. Rice: 1980, 'Slightly curved or kinked cracks'. Int. J. Frac. 16, 155-169.

Dalmas, D., E. Barthel, and D. Vandembroucq: 2009, 'Crack front pinning by design in planar heterogeneous interfaces'. J. Mech. Phys. Solids 57, 446-457.

Dalmas, D., A. Lelarge, and D. Vandembroucq: 2008, 'Crack propagation through phase-separated glasses: Effect of the characteristic size of disorder'. Phys. Rev. Lett. 101, 255501.

Dimas, L. S., G. H. Bratzel, I. Eylon, and M. Buehler: 2013, 'Tough composites inspired by mineralized natural materials: Computation, 3D printing, and testing'. Adv. Func. Mat. 23, 4629 - 4638.

Gao, H. and J. R. Rice: 1989, 'A first-order perturbation analysis of crack trapping by arrays of obstacles'. J. Appl. Mech. 56, 828-836.

Gjerden, K. G., A. Stormo, and A. Hansen: 2014, 'Universality classes in constrained crack growth'. Phys. Rev. Lett. 111, 135502.

Gjerden, K. G., A. Stormo, and A. Hansen: 2014, 'Local dynamics of a randomly pinned crack front: A numerical study'. Frontiers in Physics 2, 66.
Goehring, L., L. Mahadevan, and S. W. Morris: 2009, 'Nonequilibrium scale selection mechanism for columnar jointing'. Proc. Nat. Acad. Sci. 106, 387 - 392.

Gol'dstein, R. V. and R. L. Salganik: 1974, 'Brittle fracture of solids with arbitrary cracks'. Int. J. Frac. 10, 507-523.

Guerra, C., J. Scheibert, D. Bonamy, and D. Dalmas: 2012, 'Understanding fast macroscale fracture from microcrack post mortem patterns'. Proc. Nat. Acad. Sci. 109, 390394.

Han, K., M. Ciccotti, and S. Roux: 2010, 'Measuring nanoscale stress intensity factors with an atomic force microscope'. EPL 89, 66003.

Hansen, A. and J. Schmittbuhl: 2003, 'Origin of the universal roughness exponent of brittle fracture surfaces: stress weighted percolation in the damage zone'. Phys. Rev. Lett. 90, 045504.

Herrmann, H. and S. Roux: 1990, Statistical Models for the Fracture of Disordered Media. Elsevier.

Hild, F., A. Bouterf, and S. Roux: 2015, 'Damage measurement via DIC'. Int. J. Frac. 191, 77-105.

Hossain, M. Z., C. J. Hsueh, B. Bourdin, and K. Bhattacharya: 2014, 'Effective toughness of heterogeneous media'. J. Mech. Phys. Solids 71, 15-32.

Irwin, G. R.: 1958, 'Fracture'. In: Handbuch der Physik, Vol. 6. p. 551, Springer-Verlag.

Kachanov, M.: 2003, 'On the problem of crack interactions and crack coalescence'. Int. J. Frac. 120, 537-543.

Katzav, E., M. Adda-Bedia, and B. Derrida: 2007, 'Fracture surfaces of heterogeneous materials: A 2D solvable model'. Eur. Phys. Lett. p. 46006.

Kun, F., I. Varga, S. Lennartz-Sassinek, and I. G. Main: 2014, 'Rupture cascades in a discrete element model of a porous sedimentary rock'. Phys. Rev. Lett. 112, 165501.

Larralde, H. and R. C. Ball: 1995, 'The shape of slowly growing cracks'. Europhys. Lett. 30, 87-92.

Laurson, L., S. Santucci, and S. Zapperi: 2010, 'Avalanches and clusters in planar crack front propagation'. Phys. Rev. E 81, 046116.

Lazarus, V.: 2011, 'Perturbation approaches of a planar crack in linear elastic fracture mechanics: a review'. $J$. Mech. Phys. Solids 59, 121-144.

Legrand, L., S. Patinet, J. B. Leblond, J. Frelat, V. Lazarus, and D. Vandembroucq: 2011, 'Coplanar perturbation of a crack lying in the mid-plane of a plate'. Int. J. Frac. 170, 67-82.

Limodin, N., J. Réthoré, J. Buffière, A. Gravouil, F. Hild, and S. Roux: 2009, 'Crack closure and stress intensity factor measurements in nodular graphite cast iron using 3D correlation of lobaratory X ray microtomography images'. Acta Mater. 57, 4090 - 4101.

Maire, E. and P. J. Withers: 2014, 'Quantitative X-ray tomography'. Int. Mat. Rev. 59(1), 1-43. 
Måløy, K. J., A. Hansen, E. L. Hinrichsen, and S. Roux: 1992, 'Experimental measurements of the roughness of brittle cracks'. Phys. Rev. Lett. 68, 213-215.

Måløy, K. J., S. Santucci, J. Schmittbuhl, and R. Toussaint: 2006, 'Local waiting time fluctuations along a randomly pinned crack front'. Phys. Rev. Lett. 96, 045501.

Måløy, K. J. and J. Schmittbuhl: 2001, 'Dynamical event during slow crack propagation'. Phys. Rev. Lett. 87, 105502.

Mandelbrot, B. B., D. E. Passoja, and A. J. Paullay: 1984, 'Fractal character of fracture surfaces of metals'. Nature 308, 721-722.

Marthelot, J., B. Roman, J. Bico, J. Teisseire, D. Dalmas, and F. Melo: 2014, 'Self-replicating cracks: A collaborative fracture mode in thin films'. Phys. Rev. lett. 113, 085502.

Morel, S., D. Bonamy, L. Ponson, and E. Bouchaud: 2008, 'Transient damage spreading and anomalous scaling in mortar crack surfaces'. Phys. Rev. E 78, 016112.

Movchan, A. B., H. Gao, and J. R. Willis: 1998, 'On perturbations of plane cracks'. Int. J. Solids Struct. 35, 34193453.

Needleman, A., V. Tvergaard, and E. Bouchaud: 2012, 'Prediction of ductile fracture surface roughness scaling'. $J$. Appl. Mech. 79, 031015.

Nukala, P. K. V. V., P. Barai, S. Zapperi, M. Alava, and S. Simunovic: 2010, 'Fracture roughness in threedimensional beam lattice systems'. Phys. Rev. E 82, 026103.

Pijaudier-Cabot, G. and Z. P. Bazant: 1987, 'Nonlocal damage theory'. J. Eng. Mech. 113, 1512-1533.

Pindra, N., V. Lazarus, and J. B. Leblond: 2008, 'The deformation of the front of a 3D interface crack propagating quasistatically in a medium with random fracture properties'. J. Mech. Phys. Solids 56, 1269-1295.

Ponson, L., H. Auradou, P. Vié, and J. P. Hulin: 2006a, 'Low self-affine exponents of fractured glass ceramics surfaces'. Phys. Rev. Lett. 97, 125501.

Ponson, L., D. Bonamy, H. Auradou, G. Mourot, S. Morel, E. Bouchaud, C. Guillot, and J. Hulin: 2006b, 'Anisotropic self-affine properties of experimental fracture surfaces'. Int. J. Frac. 140, 27-36.

Ponson, L., D. Bonamy, and E. Bouchaud: 2006c, 'Twodimensional scaling properties of experimental fracture surfaces'. Phys. Rev. Lett. 96, 035506.

Ponson, L., H. Auradou, M. Pessel, V. Lazarus, and J.-P. Hulin: 2007, 'Failure mechanisms and surface roughness statistics of fractured Fontainebleau sandstone'. Phys. Rev. E 76, 036108.

Pouchou, J. L., D. Boivin, P. Beauchêne, G. L. Besnerais, and F. Vignon: 2002, '3D reconstruction of rough surfaces by SEM stereo imaging'. Microchimica Acta 139, 135144.
Ramanathan, S., D. Ertas, and D. S. Fisher: 1997, 'Quasistatic crack propagation in heterogeneous media'. Phys. Rev. Lett. 79, 873-876.

Ravi-Chandar, K. and B. Yang: 1997, 'On the role of microcracks in the dynamic fracture of brittle materials'. $J$. Mech. Phys. Solids 45, 535-563.

Réthoré, J. and R. Estevez: 2013, 'Identification of a cohesive zone model from digital images at the micron-scale'. J. Mech. Phys. Solids 61, 1407-1420.

Rice, J. R.: 1985, 'First-order variation in elastic fields due to variation in location of a planar crack front'. J. Appl. Mech. 52, 571-579.

Roux, S., D. Vandembroucq, and F. Hild: 2003, 'Effective toughness of heterogeneous brittle materials'. Eur. J. Mech. A 22, 743-749.

Santucci, S., M. Grob, R. Toussaint, J. Schmittbuhl, A. Hansen, and K. J. Måløy: 2010, 'Fracture roughness scaling: A case study on planar cracks'. EPL 92, 44001.

Santucci, S., K. J. Måløy, A. Delaplace, J. Mathiesen, A. Hansen, J. Bakke, J. Schmittbuhl, L. Vanel, and P. Ray: 2007, 'Statistics of fracture surfaces'. Phys. Rev. E 75, 016104.

Schmittbuhl, J., S. Roux, J. P. Vilotte, and K. J. Måløy: 1995, 'Interfacial crack pinning: effect of nonlocal interactions'. Phys. Rev. Lett. 74, 1787-1790.

Srivastava, A., L. Ponson, S. Osovski, E. Bouchaud, V. Tvergaard, and A. Needleman: 2014, 'Effect of inclusion density on ductile fracture toughness and roughness'. $J$. Mech. Phys. Solids 63, 62-79.

Stojanova, M., S. Santucci, L. Vanel, and O. Ramos: 2013, 'Statistical analysis of subcritical crack growth by acoustic emission vs direct imaging'. Phys. Rev. Lett.

Tallakstad, K. T., R. Toussaint, S. Santucci, and K. J. Måløy: 2013, 'Non-Gaussian nature of fracture and the survival of fat-tail exponents'. Phys. Rev. Lett. 110, 145501.

Tallakstad, K. T., R. Toussaint, S. Santucci, J. Schmittbuhl, and K. J. Måløy: 2011, 'Local dynamics of a randomly pinned crack front during creep and forced propagation: An experimental study'. Phys. Rev. E 83, 046108.

Vasoya, M., J.-B. Leblond, and L. Ponson: 2013, 'A geometrically nonlinear analysis of coplanar crack propagation in some heterogeneous medium'. Int. J. Solids Struct. 50, 371-378.

Vasoya, M., A. B. Unni, J. B. Leblond, V. Lazarus, and L. Ponson: 2015, 'A theoretical and experimental study of crack pinning by strong heterogeneities'. J. Mech. Phys. Solids 89, 211-230.

Vernède, S., L. Ponson, and J.-P. Bouchaud: 2015, 'Turbulent fracture surfaces: A footprint of damage percolation?'. Phys. Rev. Lett. 141, 215501.

Ververis, C., K. Georghiou, N. Christodoulakis, P. Santas, and R. Santas: 2004, 'Fiber dimensions, lignin and cellulose content of various plant materials and their suitability 
for paper production'. Industrial Crops and Products 19, 245-254.

Xia, S., L. Ponson, G. Ravichandran, and K. Bhattacharya: 2012, 'Toughening and asymmetry in peeling of heterogeneous adhesives'. Phys. Rev. Lett. 108, 196101. 